$11-2015$

\title{
Particle transport in low-collisionality H-mode plasmas on DIII-D
}

Saskia Mordijck

William \& Mary, smordijck@wm.edu

X. Wang

E. J. Doyle

T. L. Rhodes

et al.

Follow this and additional works at: https://scholarworks.wm.edu/aspubs

Part of the Physics Commons

\section{Recommended Citation}

Mordijck, Saskia; Wang, X.; Doyle, E. J.; Rhodes, T. L.; and et al., Particle transport in low-collisionality Hmode plasmas on DIII-D (2015). Nuclear Fusion, 55(11).

10.1088/0029-5515/55/11/113025

This Article is brought to you for free and open access by the Arts and Sciences at W\&M ScholarWorks. It has been accepted for inclusion in Arts \& Sciences Articles by an authorized administrator of W\&M ScholarWorks. For more information, please contact scholarworks@wm.edu. 


\title{
Particle transport in low-collisionality H-mode plasmas on DIII-D
}

\author{
S. Mordijck ${ }^{1}$, X. Wang ${ }^{1}$, E.J. Doyle ${ }^{2}$, T.L. Rhodes ${ }^{2}$, L. Schmitz ${ }^{2}$, L. Zeng ${ }^{2}$, \\ G.M. Staebler ${ }^{3}$, C.C. Petty ${ }^{3}$, R.J. Groebner ${ }^{3}$, W.-H. Ko ${ }^{4}$, B.A. Grierson ${ }^{5}$, \\ W.M. Solomon ${ }^{5}$, T. Tala ${ }^{6}$, A. Salmi ${ }^{6}$, C. Chrystal ${ }^{7}$, P.H. Diamond ${ }^{7}$ and \\ G.R. McKee ${ }^{8}$ \\ ${ }^{1}$ Department of Computer Science, College of William and Mary, Williamsburg, VA 23187, USA \\ 2 Department of Physics and Astronomy, University of California Los Angeles, Los Angeles, \\ CA 90095, USA \\ 3 General Atomics, PO BOX 85608, San Diego, CA 92186-5608, USA \\ ${ }^{4}$ KSTAR, National Fusion Research Institute, Daejeon, 305-806, South Korea \\ 5 Princeton Plasma Physics Laboratory, PO Box 451, Princeton, NJ 08543-0451, USA \\ 6 VTT Technical Research Centre of Finland, PO Box 1000, FI-02044 VTT, Espoo, Finland \\ 7 Department of Physics, University of California San Diego, La Jolla, CA 92093-0417, USA \\ ${ }^{8}$ Department of Engineering, University of Wisconsin, Madison, WI 53706, USA
}

E-mail: mordijck@cs.wm.edu

Received 3 March 2015, revised 27 August 2015

Accepted for publication 3 September 2015

Published 5 October 2015

\begin{abstract}
In this paper we show that changing from an ion temperature gradient (ITG) to a trapped electron mode (TEM) dominant turbulence regime (based on linear gyrokinetic simulations) results experimentally in a strong density pump-out (defined as a reduction in line-averaged density) in low collisionality, low power H-mode plasmas. We vary the turbulence drive by changing the heating from predominantly ion heated using neutral beam injection to electron heated using electron cyclotron heating, which changes the $T_{e} / T_{i}$ ratio and the temperature gradients. Perturbed gas puff experiments show an increase in transport outside $\rho=0.6$, through a strong increase in the perturbed diffusion coefficient and a decrease in the inward pinch. Linear gyrokinetic simulations with TGLF show an increase in the particle flux outside the mid-radius. In conjunction an increase in intermediate-scale length density fluctuations is observed, which indicates an increase in turbulence intensity at typical TEM wavelengths. However, although the experimental changes in particle transport agree with a change from ITG to TEM turbulence regimes, we do not observe a reduction in the core rotation at midradius, nor a rotation reversal.
\end{abstract}

Keywords: transport properties, particle flux, Tokamaks

(Some figures may appear in colour only in the online journal)

\section{Introduction}

Through control of the density gradient, particle confinement can affect the fusion power in ITER [1] and the underlying turbulent modes. Despite its importance, our understanding of particle transport lags behind heat transport. As a result, the current predictions for ITER conservatively assume a flat density profile in the core [2]. More optimistically, a peaked density profile may be obtained by operating at low collisionality [3]. However, recent work has shown that in a set of self-similarity experiments in which only collisionality was altered, no increase in density peaking was observed, in agreement with gyro-kinetic simulations [4]. So the focus is shifting from collisionality to the role of turbulence and toroidal rotation in determining particle transport [5]. In this paper we will study the effects of the underlying turbulence structure on particle transport in low torque, low collisionality H-modes on DIII-D. 
In order to study the effect of turbulence on particle transport in the DIII-D tokamak we performed a set of experiments in which the heating power is altered from predominantly ion heating (using the neutral beam injection (NBI) system) to electron heating (using electron cyclotron heating $(\mathrm{ECH})$ ). In DIII-D the ion-electron collisional coupling is weak enough that in low collisionality $\left(\nu^{*} \sim 0.2\right)$ discharges switching from ion to electron heating alters both the temperature gradients and thus the turbulence drive in the core plasma. We observe in ECH-heated plasmas a strong reduction in the density profile over the whole plasma radius, which will be referred to as 'pump-out'. In AUG experiments with the carbon wall, at low density a similar 'pump-out' was observed; however, in more recent experiments with the tungsten wall, at much higher densities/collisionalities, no such pump-out is observed [5, 6]. In these higher density/collisionality plasmas, overall confinement increases along with an increase in the peaking of the density at mid-radius.

Turbulence in a tokamak plasma is gradient driven. In predominantly ion heated plasmas, the ion temperature gradient (ITG) drive will be dominant, whereas in plasmas that are dominantly electron heated, the electron temperature gradient becomes the driving force. This results in a switch to trapped electron mode (TEM) driven turbulence. The TEM mode depends on the electron temperature gradient as well as the electron density gradient. Theory predicts that changing from dominantly ITG to TEM turbulence regimes results in the reversal of the group velocity of the turbulent structures from the ion to the electron direction [7]. This will then result in the reversal of the intrinsic rotation component of the toroidal rotation, which in a torque-free plasma leads to a rotation reversal of the toroidal rotation [7]. The sign change of the turbulence group velocity also changes the thermodiffusion component of the particle flux from an inward (ITG) to outward flux (TEM) [8]. There is a large body of work that links toroidal rotation reversals with linear ITG-TEM transitions; however, more experimental observations find that rotation reversals can also occur without a change in local gradients and thus without a change in the calculated linear turbulence regime [9-13]. With relation to particle transport, not only does the sign change from ITG to TEM matter, but also the frequency of the mode plays an important role [5].

In this paper we observe a change in linear stability from ITG to TEM, but we do not observe a rotation reversal. We do, however, observe a change in particle transport and an overall reduction in the density profile from particle balance analysis. Perturbed gas puff experiments show an increase in the particle transport outside $\rho \sim 0.6$ (with $\rho$ being defined as the square root of the poloidal flux). This is in agreement with quasi-linear modeling using the TGLF transport model, which shows that particle transport does not change much around mid-radius, but increases significantly at $\rho \sim 0.74$ in the ECH heated plasmas. Mid-scale density fluctuations increase from mid-radius and outward for the TEM unstable plasmas. All these results point to a strong increase in particle transport in the outer half of the plasma. In the discussion we will compare our results to previous results from other tokamaks with relation to particle transport.

\section{Experimental setup}

These experiments in DIII-D [14] focus on low power (3-4 MW) low normalised plasma pressure, $\beta_{N} \sim 1-1.5 \mathrm{H}$-mode plasmas in the ITER Similar Shape (ISS) at a $q_{95} \sim 4.2$, plasma current, $I_{\mathrm{p}}=1.1 \mathrm{MA}$, toroidal magnetic field, $B_{\mathrm{T}}=1.9 \mathrm{~T}$, with zero and low co-torque $(0.5 \mathrm{~N} \mathrm{~m})$ injection from the NBI system. This allows us to avoid locked modes and still operate at a low line averaged electron density $n_{e} \sim 2-4 \times 10^{19} \mathrm{~m}^{-3}$. This results in a collisionality of $\nu^{*}=q_{95} R \epsilon^{-3 / 2} \lambda_{e}^{-1} \sim 0.2$ -0.5 at the top of the pedestal. Here, $R$ is the major radius, $\epsilon(\equiv a / R)$ is the inverse aspect ratio, $a$ is the minor radius, $q_{95}$ is the safety factor at the $95 \%$ flux surface and $\lambda_{e}=v_{T_{e}} \tau_{e}$ is the product of electron thermal velocity $v_{T_{e}}$ and electron-electron collision time $\tau_{e}$. At $2000 \mathrm{~ms}$, we reduce the initial torque input from the neutral beams towards a balanced beam input, while keeping the NBI beam input power close to $3 \mathrm{MW}$ (figures 1 and 2). The experiments presented in this paper were performed on different days and we show the time evolution of the main characteristics in two separate figures to highlight that the plasma conditions and input parameters were similar (figures 1 and 2) on both experimental days. In order to increase the ratio of electron temperature over ion temperature $\left(T_{e} / T_{i}\right)$, we replace part of the NBI heating (black time trace) with $\mathrm{ECH}$ in separate discharges (red and blue time traces). Using $\mathrm{ECH}$ increases $T_{e}$ in the plasma core, which in turn increases the electron temperature gradient, $1 / L_{T_{e}}$. This results in a strong reduction of the line averaged density, and in order to avoid a back transition to the L-mode, we have to add a gas puff, depending on the wall conditions (figures 1 and 2).

Applying $\mathrm{ECH}$ heating in these low density plasmas allows us to increase the $T_{e} / T_{i}$ ratio from 0.7 to 2.0 (figure $3(a)$ ). Previous work in AUG has shown an increase in density peaking with an increased $T_{e} / T_{i}$ ratio $[5,10]$. In these DIII-D $\mathrm{H}$-mode plasmas, we have not observed such a trend (figure 4). Using the reflectometer to measure the electron density, we observe a strong overall reduction in the density profile (figure $3(b)$ ). The density loss is not just located at the plasma edge, but also in the plasma core. However, in order to make a prediction as to how the particle transport changes, we first need to address the changes in particle balance, owing to changes in the NBI core fueling.

In order to assess whether the changes in the density profile are purely the result of a change in fueling, we need to look at the particle balance $\frac{\partial n}{\partial t}=-\nabla \cdot \Gamma+S$, where $n$ is the density, $t$ is the time, $\Gamma$ is the particle flux and $S$ is the particle source. In figure 1, the ECH heated plasma received an additional gas puff to maintain H-mode confinement, while the NBI fueling is reduced by $50 \%$. In figure 2 , no such increase in gas puff is needed to keep the ECH heated plasma in H-mode, due to slightly different wall conditions in comparison with the discharges in figure 1. Not accounting for the amount of fueling from the walls, which is difficult to assess, especially without any information on the amount of particles being lost to the cryo-pump, the amount of fueling in the ECH heated plasma was $50 \%$ higher $\left(12\right.$ TorrL s$^{-1}$ ) versus the NBI heated plasmas $\left(8\right.$ TorrL $\left.\mathrm{s}^{-1}\right)$ in the worst case example shown in 


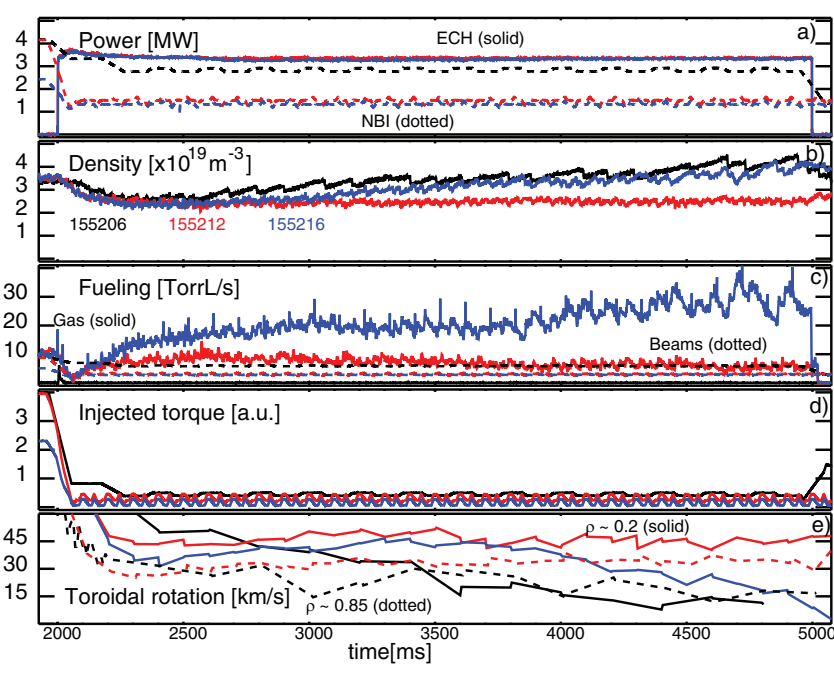

Figure 1. Time evolution of three representative discharges in which the heating was changed from (a) NBI (dashed lines) to ECH (solid). (b) Shows the evolution of the density of the ECH (red and blue) and the NBI (black) heated discharges. In order to limit the density reduction for the ECH discharge, a gas puff is added (red) in another ECH discharge a gas puff is added to match the density from the NBI heated discharge $(c)$. (d) Shows the injected torque for both discharges and $(e)$ has the resulting core (solid) and edge (dashed) carbon toroidal rotation.

figure 1. At the same time there is a reduction in the line averaged density from $3.5 \times 10^{19} \mathrm{~m}^{-3}$ in the NBI-heated plasma to $2.0 \times 10^{19} \mathrm{~m}^{-3}$ for the ECH-heated plasma. The combination of increased fueling and reduced density means that the overall particle transport increases.

Assessing how fueling from the wall changes in these discharges is difficult. The strike-point is in the optimal location for pumping, but without pressure measurements in the pump plenum it is not possible to assess where the outflux of particles ends up. There is no increase in $D_{\alpha}$ light in the divertor area, which is an indication that recycling in this area does not increase. Examining the extremes from where the gas puff fueling (gas valve is located close to the top of the plasma on the low field side) does not fuel the plasma to where the gas puff fully fuels the plasma, this leads to an increase in outward particle flux from $7 \%$ to $60 \%$ at the edge. In other similar plasmas, in which we did not need to add an additional gas puff (figure 2), a gas balance assessment using the same limitations as described in this paragraph results in a $33 \%$ increase in particle flux at the plasma edge.

Another way to approach this problem of particle balance is to look at the changes in particle flux $\Gamma$ (figure 5(a)) and compare these to changes in the density gradient. We observe a $50 \%$ reduction of the particle flux as calculated by ONETWO, which is a direct consequence of the $50 \%$ reduction in the core fueling. ONETWO does not have a reliable model to capture the effects of edge fueling, so the results closer to the plasma edge will not correctly capture the changes in experimental particle flux. If we assume that the effective diffusion coefficient $D$ does not change, then all the changes in $\Gamma=-D \frac{\partial n}{\partial r}$ are the result from a change density gradient. Figure $5(b)$ shows that the density gradient is not reduced by $50 \%$ over the plasma core. There is a

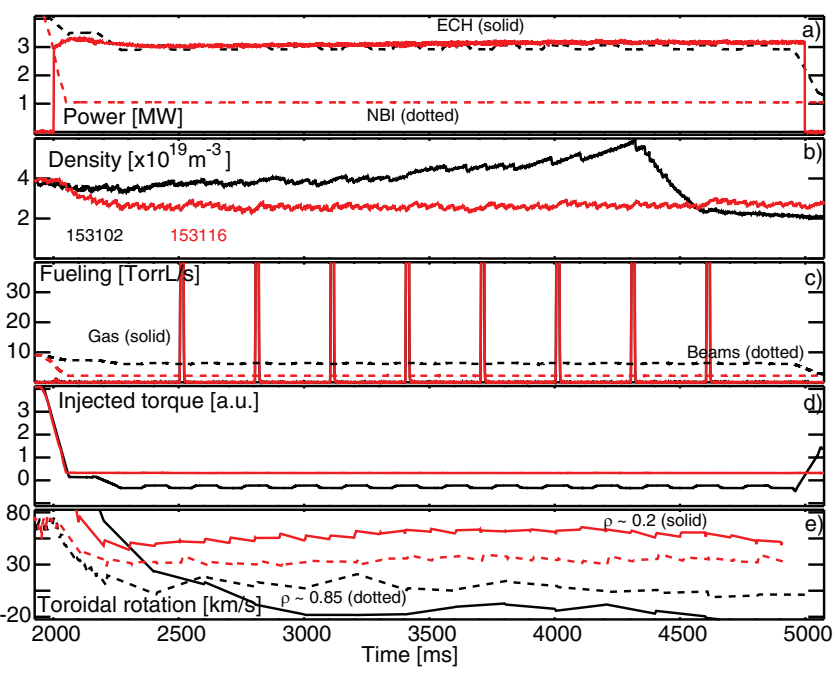

Figure 2. Time evolution of two representative discharges in which the heating was changed from $(a) \mathrm{NBI}$ (dashed lines) to ECH (solid). (b) Shows the evolution of the density of the ECH (red) and the NBI (black) heated discharges. In order to limit the density reduction for the ECH discharge, a gas puff is added $(c)$. (d) Shows the injected torque for both discharges and $(e)$ has the resulting core (solid) and edge (dashed) carbon toroidal rotation.

very strong reduction close to the magnetic axis by about $75 \%$, but close to mid-radius, both gradients are very similar. As a result, $D$, the effective diffusion coefficient will not be the same in the ECH-heated discharge and the NBI-heated discharge (figure $5(c)$ ). This shows that the changes in the density profile are the result of changes in transport, not just the result of changes in core fueling and that these changes in transport are different at different radial locations.

As a final measure for the changes in transport, figure 6 shows $1 / L_{N}=\nabla n / n$ as a function of radius for an NBI-heated discharge versus an ECH-heated discharge. $\nabla n / n$ is often used as a measure for the ratio of inward pinch over outward diffusion $v / D$ (this is assuming that fueling is negligible). From $\rho \sim 0.3$ and outward, there is nearly no difference between the ECH-heated discharge and the NBI-heated discharge. This would suggest that the ratio of $v / D$ does not change, but the individual components could change. In the next section we will look more closely at the measured changes in particle transport using a perturbative technique.

\section{Changes in particle transport}

In order to assess the changes in particle transport, we use a perturbative gas puff technique. This technique has been successfully used to address the changes in particle transport as a result of resonant magnetic perturbations (RMPs) and collisionality in DIII-D plasmas [4, 15]. Other devices, such as LHD and JET have also recently used the mathematical derivation by Takenaga et al [16] to study particle transport [17, 18]. By applying a small perturbative gas puff, we modulate the density profile with a fixed modulation frequency. Using Fourier analysis we can extract the phase and amplitude of this modulation. Fourier decomposing the perturbed continuity 

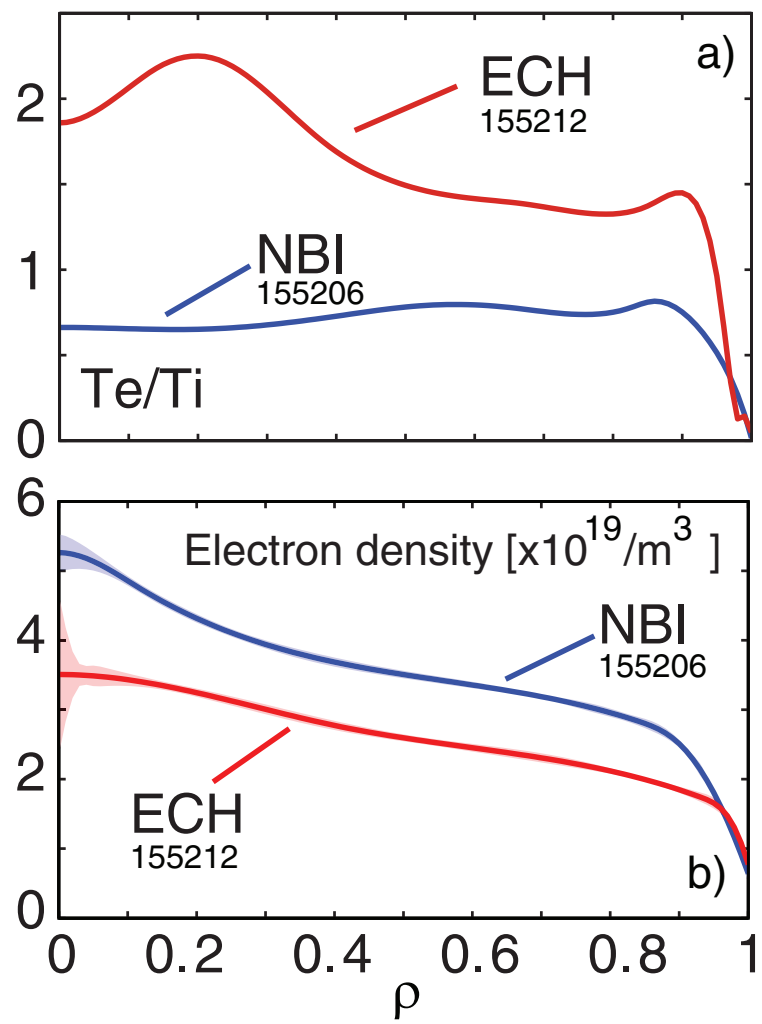

Figure 3. (a) $T_{e} / T_{i}$ ratio for an ECH-heated discharge (red) and an NBI-heated discharge (blue) versus $\rho$. These curves are based on polynomial fits to the ion temperature measured with CER and the electron temperature measured with Thomson scattering as well as ECE. (b) The electron density polynomial profile fits using the profile reflectometry as a function of $\rho$. A strong reduction in the overall density profile is observed in the ECH-heated (red) plasma in comparison to the NBI-heated plasma (blue).

equation, the perturbed diffusion coefficient, $D_{\mathrm{p}}$ and the perturbed convection coefficient, $v_{\mathrm{p}}$ can be expressed in terms of this amplitude and phase. We refer the reader to these previous publications and the original paper by Takenaga et al [16] as well as early work in the 1990s on perturbative transport techniques for the equations and the full derivation [19-22]. Using this technique we can extract the perturbed transport coefficients for the NBI-heated plasmas as well as the ECH heated plasmas (figure 7). Figure 7 shows that the changes in transport are not just restricted to the diffusion coefficient, $D_{\mathrm{p}}$, but also affect the inward pinch, $v_{\mathrm{p}}$ (with a negative value pointing to an inward pinch). Up to $\rho=0.5$ the changes in $D_{\mathrm{p}}$ and $v_{\mathrm{p}}$ of the ECH-heated plasma in comparison to the NBI-heated plasma are small and mostly restricted to a small increase in $D_{\mathrm{p}}$. From $\rho=0.5-0.65$ the more pronounced increase in outward diffusion with ECH is countered by a strong increase in a inward pinch. Outside $\rho=0.65$, the strong increase in outward diffusion and the reduction in the inward pinch, result in a strong increase in outward transport in the ECH-heated plasmas, as expressed by the $v_{\mathrm{p}} / D_{\mathrm{p}}$ fraction. We only show the result up until $\rho=0.8$, because the perturbative transport analysis does not yet include the perturbative neutral fueling source, which will strongly affect the results at the plasma edge.

When we compare the $v_{\mathrm{p}} / D_{\mathrm{p}}$ ratios directly with the inverse density gradients we observe that the $v_{\mathrm{p}} / D_{\mathrm{p}}$ ratio for $\rho=0.1$

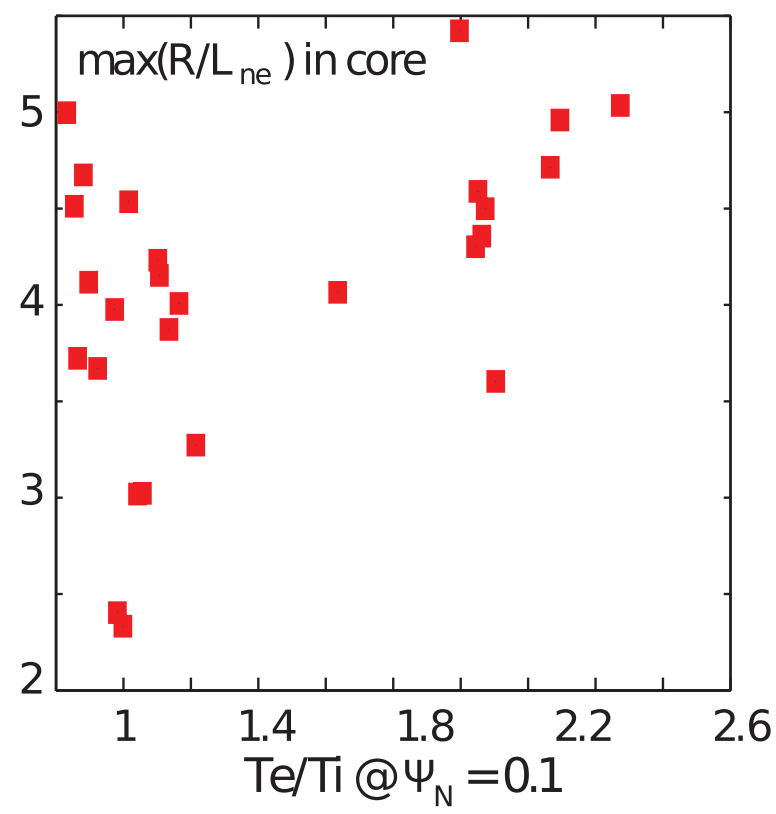

Figure 4. Database of discharges in which the $T_{e} / T_{i}$ ratio was varied by adding $\mathrm{ECH}$ heating as a function of the maximum inverse density gradient $\left(1 / L_{n}\right)$ in the core plasma. The radial locaition of the maximum inverse density gradient can vary.

-0.5 is somewhere between -1.0 and $0.1 \mathrm{~m}^{-1}$, see figure $8(a)$. The inverse density gradients on the other hand for the NBI heated discharge ranges from -2 to $-1.1 \mathrm{~m}^{-1}$ over the same radii. The direct comparison of $1 / L_{n}$ with $v_{\mathrm{p}} / D_{\mathrm{p}}$ is only valid when there is no fueling and no changes over time in line averaged density:

$v_{\mathrm{p}} / D_{\mathrm{p}}=1 / L_{n}+\frac{1}{n D_{\mathrm{p}}} \frac{1}{\mathrm{Vol}^{\prime}} \int S \mathrm{dVol}-\frac{1}{n D_{\mathrm{p}}} \frac{1}{\mathrm{Vol}^{\prime}} \int \frac{\partial n}{\partial t} \mathrm{dVol}$

Here $S$ represents the source and Vol is the plasma volume. With these plasmas being fueled by the NBI, we need to add the contribution of the fueling to the comparison and as can be observed in figure 2, there is a slow rise in the line averaged density for the NBI heated discharge, which needs to be taken into account in the comparison. Adding the source term (labeled $S_{\mathrm{T}}$ in the figures), see figure 8 , improves the comparison especially for the NBI heated discharge in the core (where fueling is a factor 2 higher for the NBI heated plasma than the ECH heated plasma). We are using the source from the ONETWO calculation, which does not have a good model for the source from the plasma edge, which limits to applicability of using this model outside the plasma core (inside $\rho=0.5$ ). In order to calculate $\frac{\partial n}{\partial t}$ we used a linear regression $n=a_{1} t+a_{0}$ to fit the time evolution of the density as measured with the reflectometer at each radial location and averaged over the time interval of the gas puff modulation, so that $\frac{\partial n}{\partial t}=a_{1}$. The addition of this term (labeled $n_{t}$ in the figures) provides a small correction for the NBI heated discharge and is negligible for the ECH heated discharge. The inclusion of these terms improves the comparison of the perturbed transport coefficients with the steady-state profiles for $\rho=0.1-0.5$. 

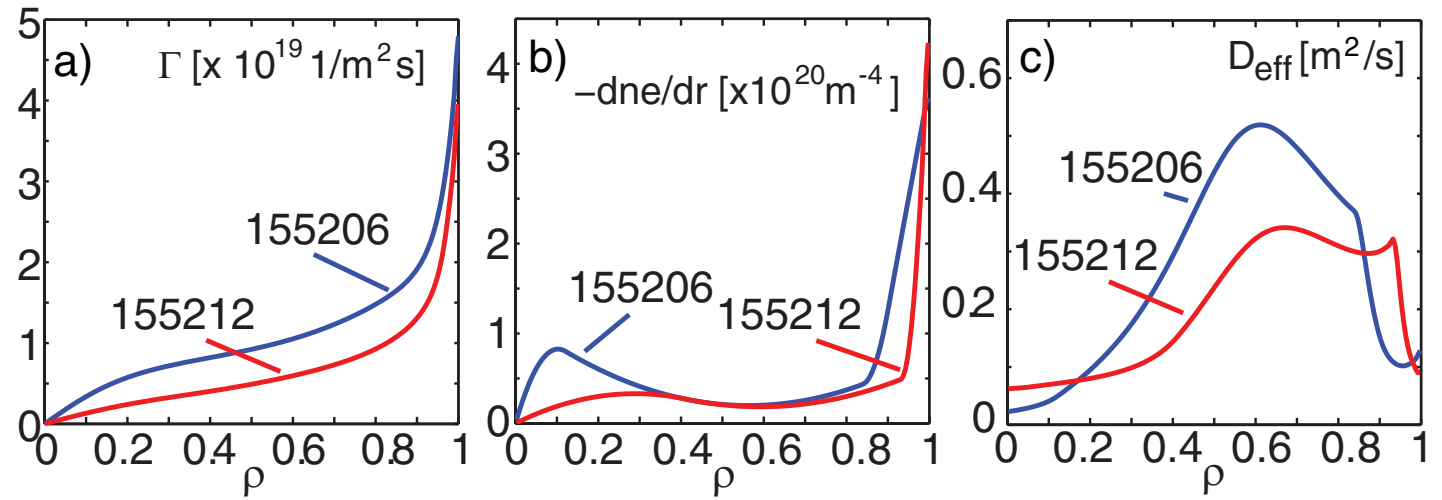

Figure 5. (a) Particle flux calculated with ONETWO for an ECH (red) and a NBI (blue) heated plasma. The particle flux is over the core region about a factor 2 lower for the ECH-heated discharge, due to a lower core source from the neutral beams. $(b)$ The density gradient for the same ECH (red) and NBI (blue) discharge does not show the same average factor 2 decrease in the core. (c) The effective diffusion coefficient based on the ONETWO flux and the experimental density gradient for the NBI (blue) and ECH (red) discharge.

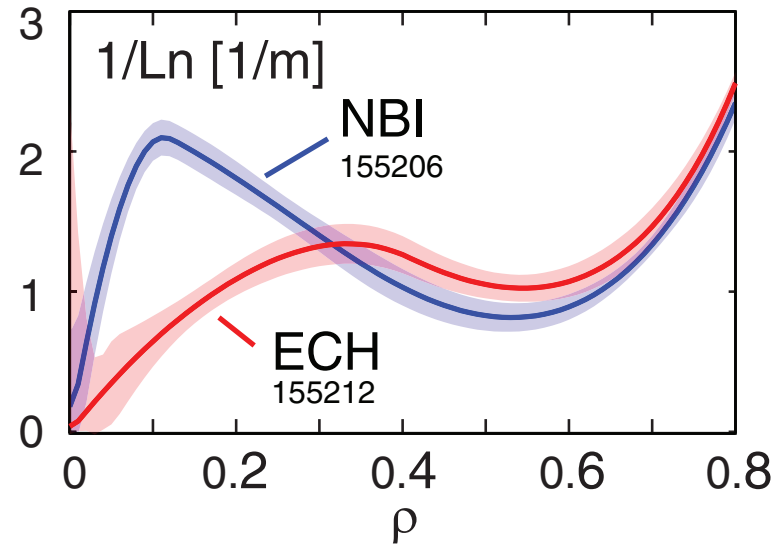

Figure 6. Inverse density gradient, $1 / L_{N}$, as a function of $\rho$ shows that the NBI (blue) heated discharge is much more peaked in the core, due to core fueling from the beams. The ECH (red) heated discharge is more peaked outside $\rho \sim 0.3$.

There is still a substantial difference for the NBI heated discharge for $\rho=0.1-0.2$. This might be the result of the fact that we have to use $D_{\mathrm{p}}$ as a proxy for the steady-state diffusion coefficient, $D$. Also, a closer look at the fueling model in ONETWO might be merited. Moreover, the gas puff perturbs the density profiles and depending on the gas puff size, as well as background transport and the radial location this can be on the order of $15 \%$ (the goal is to minimise the perturbation, while still obtaining good enough data to extract an amplitude and phase from the Fourier analysis). The amplitude of the perturbation is not constant across the plasma radius, which means that the local density gradients are affected. The 'cold' gas puff will also affect the electron temperature, which will affect the electron temperature gradient. It is not yet clear how much these changes in local gradients will affect the background transport. Recent results from JET show that when the size of the gas puff is doubled, the amplitude doubles, while the phase is not affected [23]. This would indicate that the changes in local gradients do not affect the perturbed transport coefficients and that the perturbative coefficients might be very similar to the background transport coefficients in certain plasma regimes.

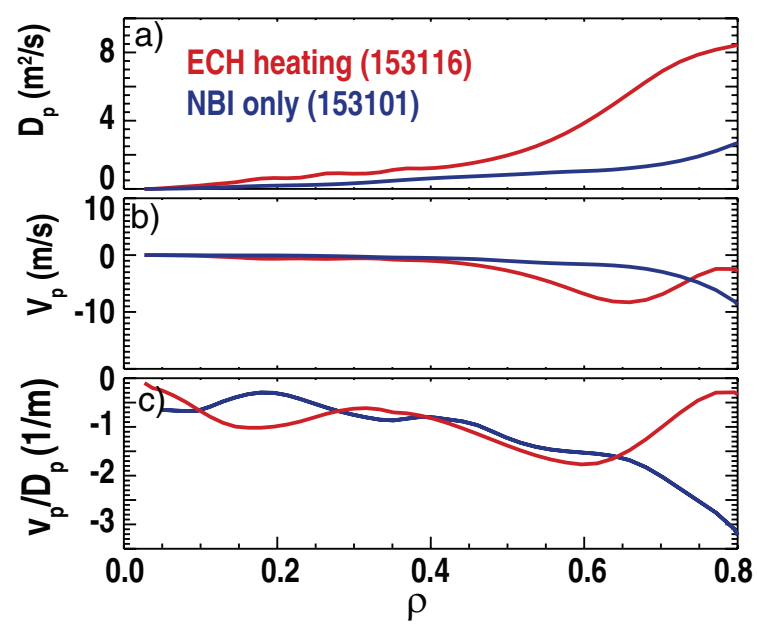

Figure 7. Perturbed transport coefficients obtained using a perturbative gas puff. (a) The perturbative diffusion coefficient increases strongly outside mid-radius for the $\mathrm{ECH}$ (red) heated plasma versus the NBI (blue) heated plasma. (b) Part of the increase in outward diffusion is countered by an increase in inward pinch just outside mid-radius in the ECH-heated plasmas. (c) The $v_{\mathrm{p}} / D_{\mathrm{p}}$ ratio shows that the increase in transport for the ECH-heated plasma occurs mostly outside $\rho \sim 0.6$.

In order to compare these perturbative transport results with turbulent particle transport we use the trapped gyro-landau fluid (TGLF) transport code. TGLF is an eigenvalue code with turbulence saturation rules that solves the gyro-Landau fluid equations and includes the effects of shaped geometry via the Miller equilibrium model [24]. Also included are trapped particle physics, collisions, ExB shear; the code spans ITG/TEM wavelengths as well as short wavelength ETG modes. Figure 9 shows the particle flux as a function of $k_{\theta} \rho_{s}$, where $k_{\theta}$ is the wave number and $\rho_{s}$ is the ion sound gyro radius. The turbulent particle flux is calculated in a similar way as the linear growth rates are calculated and based on experimental profiles as input. Integrating over $k_{\theta} \rho_{s}$, shows that although the peak in the normalised particle flux has shifted to higher $k_{\theta} \rho_{s}$ in the ECHheated plasmas at $\rho=0.5$, the integrated particle fluxes for the $\mathrm{ECH}$ and NBI cases are similar. Further out at $\rho=0.74$, there is a large increase in normalised particle flux for the $\mathrm{ECH}$-heated 

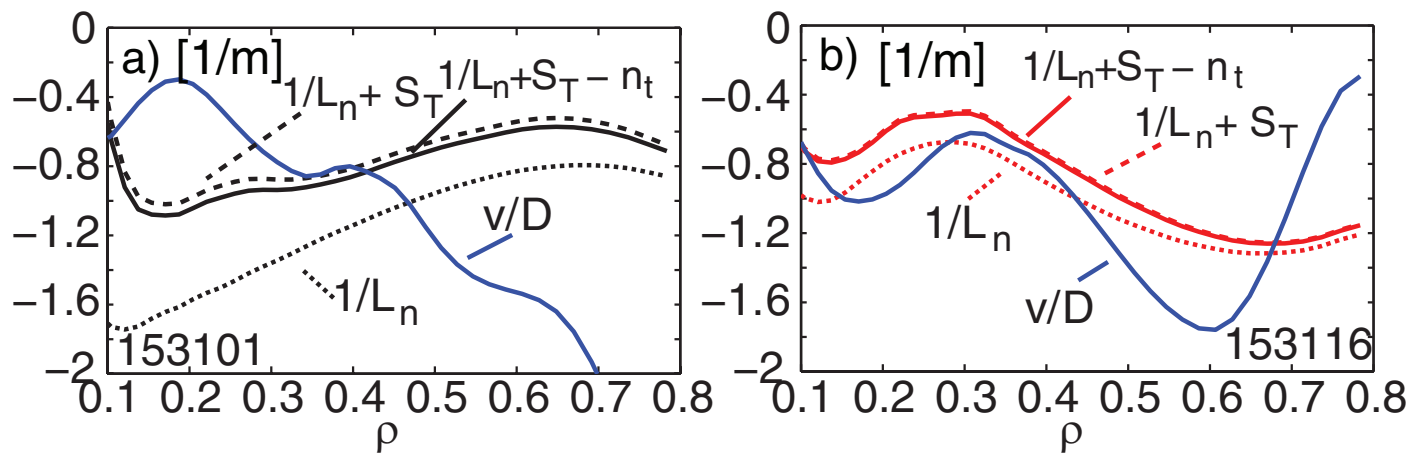

Figure 8. Comparison of $v_{\mathrm{p}} / D_{\mathrm{p}}$ ratio with the inverse density gradient $1 / L_{n}$ for an NBI heated discharge $(a)$ and an ECH heated discharge $(b)$. When the effects of the NBI fueling $\left(S_{\mathrm{T}}\right)$, see equation (1) are added to the $1 / L_{n}$ along with the slow changes in the density $\left(n_{t}\right)$, the agreement between the steady-state and perturbed transport coefficients improves for both discharges.

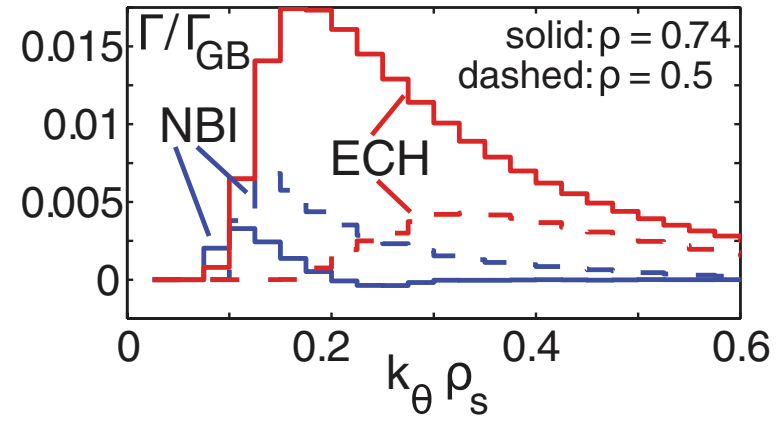

Figure 9. Calculated $\Gamma / \Gamma_{\mathrm{GB}}$ using TGLF for NBI-heated (blue) and ECH-heated (red) plasma at two radial locations $\rho=0.5$ (dashed) and $\rho=0.74$ (solid). The particle flux moves to higher $k_{\theta} \rho_{s}$ for the ECHheated plasma, but the integrated flux only increases at $\rho=0.74$.

plasmas in comparison with the NBI-heated plasmas. This observation is not in agreement with the experimental changes in the perturbed $D_{\mathrm{p}}$ and $v_{\mathrm{p}}$ transport coefficients, nor with the increase in steady-state particle flux. This motivates us to look more closely at the changes in turbulence in the next section.

\section{Turbulence changes}

To better understand how macroscopic transport changes in these plasmas we have to look at the changes in turbulence characteristics. From figure 3(a) we can see that changing the heating from NBI to $\mathrm{ECH}$ changes the $T_{e} / T_{i}$ ratio. As a result, in these low collisionality ECH-heated plasmas the turbulence regime will be dominated by the electron temperature gradient. Figure 10 shows that the frequency of the largest growth rate changes from the ion to the electron direction in the ECH-heated plasmas, whereas for the NBI-heated experiments the mode frequency remains in the ion direction. This is an indication that, based on linear stability analysis, the plasma switches from being ITG dominant to TEM dominant close to mid-radius. This switch from the ion to the electron direction will change the thermo-diffusion component of the turbulent particle flux from inward to outward. The radial location where we observe the changes in turbulence is in agreement with the radial location where the perturbative diffusion and pinch coefficients have the largest changes, even if the $v_{\mathrm{p}} / D_{\mathrm{p}}$ ratio is unaffected.

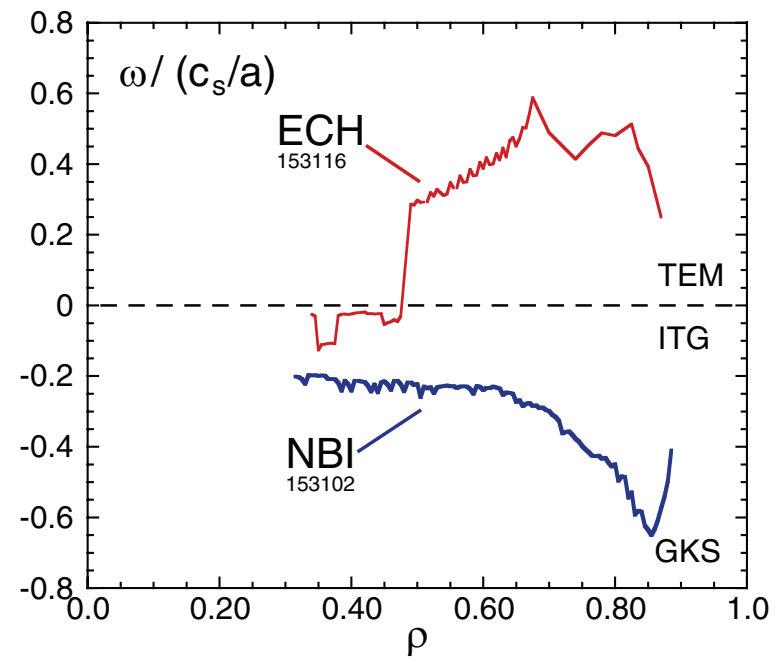

Figure 10. Frequency of the largest growth rate using GKS gyrokinetic code [27] shows a cross-over from ITG to TEM for the ECH-heated (red) discharge close to mid-radius. The NBI-heated (blue) discharge remains in the ITG regime over the whole radius.

More detailed linear gyrokinetic simulations with TGLF from $\rho=0.35-0.75$ show that in the case of the NBI heated plasmas, the peak of the linear growth rate is close to $k_{\theta} \rho_{s} \sim 0.35$ (figure $11(a)$ ). Outside $\rho=0.7$, there is a clear shift to higher $k_{\theta} \rho_{s}$ values. At the same time, we find that the frequency of these modes is in the ion direction for $\rho<0.7$ and in the electron direction further out (figure 11(c)). For the ECH heated discharges, the largest growth rates occur at $k_{\theta} \rho_{s} \sim 1.2$ (figure $11(b)$ ). The maximum value of the linear growth rates for the ECH heated discharge is about twice the value of that of the NBI heated discharge. As a result the linear growth rates for $k_{\theta} \rho_{s} \sim 0.5-1$ are of the same magnitude in both discharges. In the ECH heated plasmas, the frequency of the modes is in the electron direction from $\rho=0.47-0.75$.

Experimentally, there is an increase in mid-range $k_{\theta} \rho_{s} \sim 1.5$ -3 density fluctuations in the ECH heated plasmas in comparison with the NBI heated plasmas as measured with the Doppler Backscattering (DBS) [25], as seen in figure 12. This increase in mid-range fluctuations can mean that there is a shift to higher $k_{\theta} \rho_{s}$ turbulent structures, or that there is a global increase in turbulence. Without fluctuation measurements at 

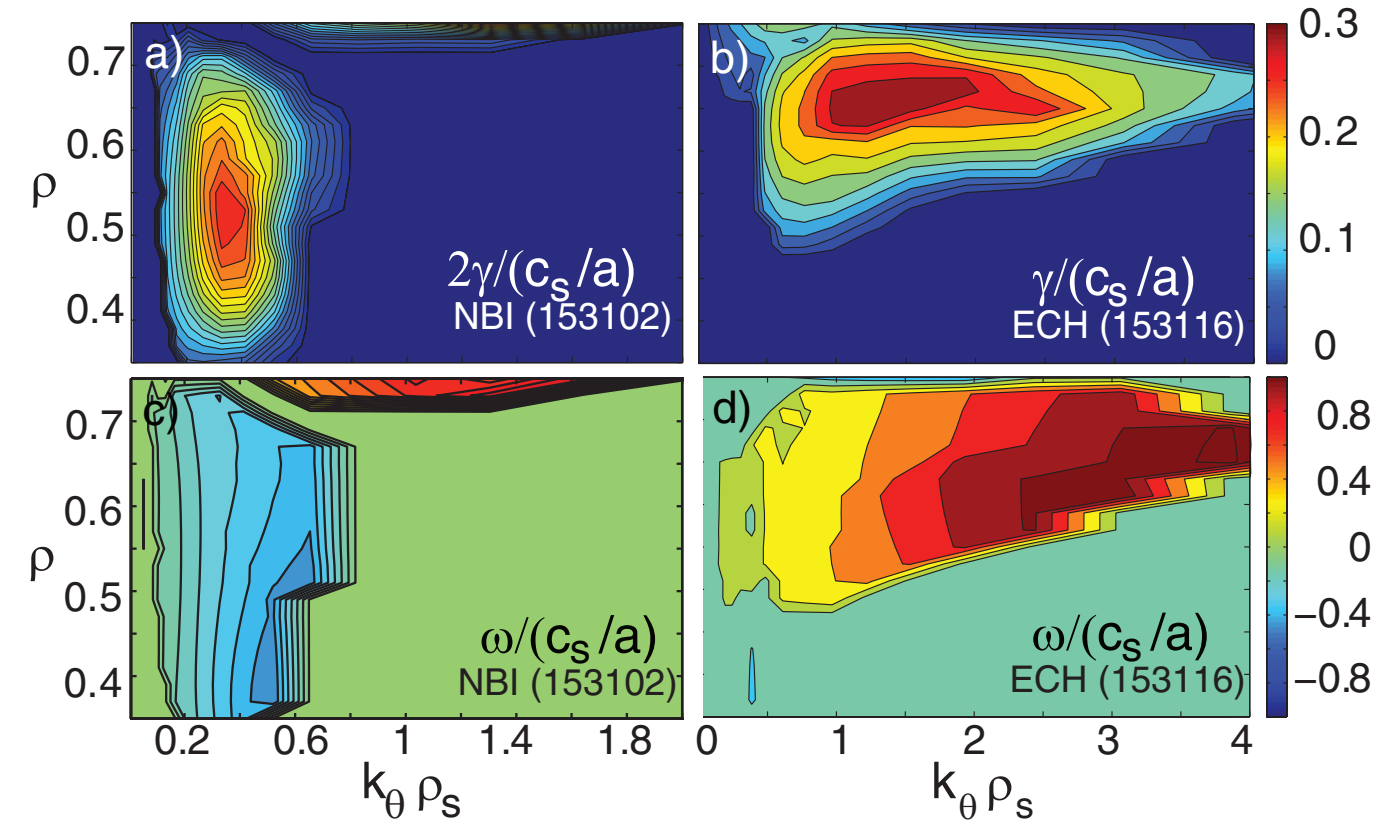

Figure 11. Contour plots showing the linear growth rate $((a)$ and $(c))$ and the corresponding frequency $((b)$ and $(d))$ using TGLF for an NBI and an ECH heated discharge from $\rho=0.35-75$ for low and intermediate $k_{\theta} \rho_{s}$ scales. The maximum linear growth rate in the ECH heated plasma is twice that of an NBI heated discharge and has shifted to higher $k_{\theta} \rho_{s}$. The frequency for the ECH heated discharge is in the electron direction from $\rho=0.5$ for all $k_{\theta} \rho_{s}$, whereas the frequency is in the ion direction for all $k_{\theta} \rho_{s}$ for the NBI heated discharge.

different wave-lengths, it is impossible to assess experimentally whether this is a global increase in turbulence, or whether this is a shift to higher $k_{\theta} \rho_{s}$.

\section{Discussion}

In this paper we have shown that by increasing the $T_{e} / T_{i}$ ratio, along with the local temperature gradients, we get a strong density pump-out. This increase in $T_{e} / T_{i}$ changes the linear stability from dominantly ITG in the NBI-heated plasmas to dominantly TEM in the ECH-heated plasmas. The local changes in density peaking and thus particle transport do not only depend on the sign change of the mode velocity, but also on the frequency of the mode. Maximum peaking is observed when the frequency reaches zero and then decreases rapidly when crossing over to the TEM regime [5]. However, the peaking of the density close to mid-radius is independent of the strong reduction in the line-averaged density. In AUG, the density pump-out was observed at low collisionality/density before the installation of the metallic wall, similar to the results presented in this paper [6]. However, with the metallic wall and operation at higher densities/collisionalities, AUG does not observe a density pump-out and the changes in the density gradient at mid-radius result in an overall improvement in particle confinement [5]. Moreover, recent results on JET with the new ITER Like Wall (ILW) show that the new wall not only affects the pedestal density, but also changes core density gradients [26]. This is an indication that the role of the first wall material can play a role in determining the overall particle confinement. A more careful database like analysis of the DIII-D experiments is needed to make a more direct comparison to the AUG results.

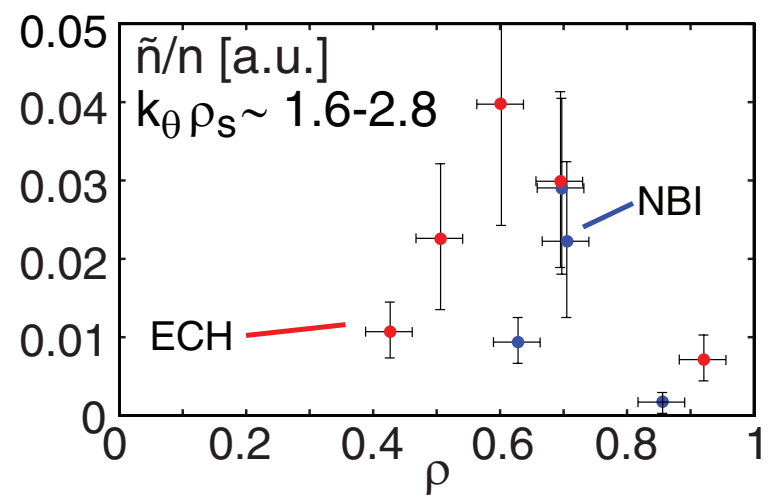

Figure 12. Intermediate scale density fluctuations measured with the DBS for an ECH-heated (red) versus an NBI-heated (blue) discharge. There is an increase in $\tilde{n} / n$ from mid-radius outward for the ECH-heated discharge, which is an indication of an increase in TEM size turbulence.

Another important observation is that although linear gyrokinetic simulations show that we transition from an ITG to a TEM-dominated regime around mid-radius, the typical rotation reversal or even a strong reduction in the toroidal rotation is not observed (figure 13). The only changes in toroidal rotation are in the plasma core and in the plasma edge. The change in the edge rotation was observed in every discharge in which ECH was applied, whereas the change in the core rotation did not follow a clear trend. Adding a gas puff in the $\mathrm{ECH}$ heated plasmas to restore the pedestal density of the NBI-heated plasmas, resulted in the return of the 'well' in the edge toroidal rotation. As a result, one could question whether the plasma actually transitions from ITG to TEM. However, recent publications with relation to the rotation reversal show that they do not solely depend on local linear ITG-TEM transitions [11, 12]. 


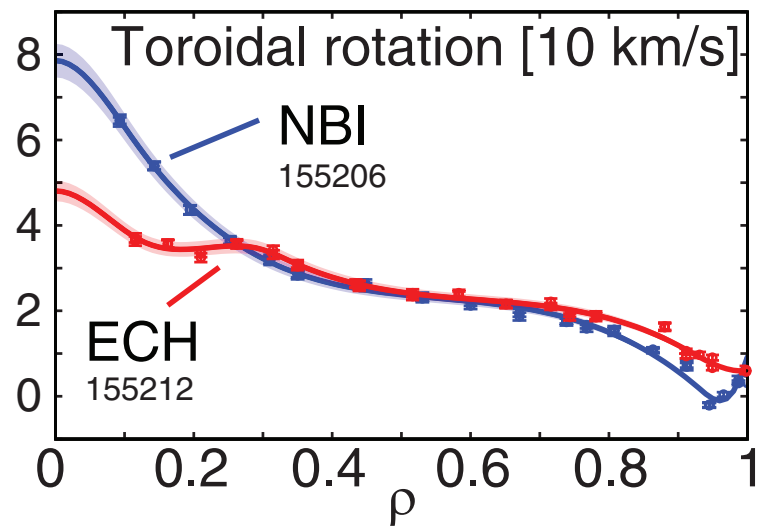

Figure 13. Carbon impurity toroidal rotation profiles as a function of $\rho$ for an ECH-heated (red) and an NBI-heated (blue) discharge. No rotation reversal is observed, only a flattening of the profile in the core and the 'well' in the edge rotation disappears with ECH heating.

Moreover, in AUG these ITG-TEM transitions also result in an increase in density peaking. We did not observe an increase in density peaking and especially not an increase in particle confinement, which would be beneficial to ITER and future tokamaks. In DIII-D, a transition from ITG to TEM does not increase the peaking and due to a large increase in transport outside mid-radius, global particle confinement is reduced.

\section{Conclusion}

In this paper we show that a change in turbulence regime (based on linear gyrokinetic simulations) from ITG to TEM results in a strong reduction in line-averaged density. The reduction in the density cannot be explained by a reduction in core fueling. There is a weak dependence on density peaking with increasing $T_{e} / T_{i}$. This is in agreement with theory, which predicts an increase in outward particle flux when the turbulence group velocity changes sign. TGLF simulations as well as perturbed transport coefficients show a strong increase in particle transport outside $\rho=0.6$. We find that in the region in which linear stability predicts a change from ITG to TEM, there is a strong increase in the diffusion coefficient. A comparison between $v_{\mathrm{p}} / D_{\mathrm{p}}$ and the inverse density scale length shows the importance of including the effects of the neutral beam fueling source as well as the slow density evolution. Intermediate scale fluctuations show a strong increase outside $\rho=0.6$, as might be expected when the plasma transitions to the TEM regime. We do not observe the typical rotation reversal or reduction in the toroidal rotation associated with ITG-TEM transitions. Future work will focus on understanding the role of the toroidal rotation gradient and collisionality on particle transport. In order to better understand how these turbulence changes affect toroidal rotation, we will compare perturbative beam modulations with changes in momentum transport.

\section{Acknowledgments}

This material is based on work supported by the U.S. Department of Energy, the Office of Science, the Office of Fusion Energy Sciences, using the DIII-D National
Fusion Facility, a DOE Office of Science user facility, under Awards DE-FC02-04ER54698, DE-SC0007880, DE-FG0208ER54984, DE-AC02-09CH11466, DE-FG02-07ER54917, DE-FG02-89ER53296, and DE-FG02-08ER54999. DIII-D data shown in this paper can be obtained in digital format by following the links at https://fusion.gat.com/global/D3D_ DMP. We would like to thank Pieter Peers for childcare assistance to make these experiments possible and Mumford and Sons for providing the soundtrack.

\section{References}

[1] Hawryluk R.J. et al 2009 Nucl. Fusion 49065012

[2] Garzotti L. et al 2012 Nucl. Fusion 52013002

[3] Angioni C., Peeters A.G., Pereverzev G.V., Ryter F., Tardini G. and ASDEX Upgrade Team 2003 Phys. Rev. Lett. 90205003

[4] Doyle E.J. et al 2012 Particle transport results from collisionality scans and perturbative experiments on DIII-D Proc. 24th Int. Conf. on Fusion Energy Conf. (San Diego) www-naweb.iaea.org/napc/physics/FEC/FEC2012/ papers/376_EXP326.pdf

[5] Angioni C., Camenen Y., Casson F.J., Fable E., McDermott R.M., Peeters A.G. and Rice J.E. $2012 \mathrm{Nucl}$. Fusion $\mathbf{5 2} 114003$

[6] Angioni C., Peeters A.G., Garbet X., Manini A., Ryter F. and the ASDEX Upgrade Team 2004 Nucl. Fusion 44827

[7] Diamond P.H. et al 2013 Nucl. Fusion 53104019

[8] Garbet X., Garzotti L., Mantica P., Nordman H., Valovic M., Weisen H. and Angioni C. 2003 Phys. Rev. Lett. 91035001

[9] Bortolon A., Duval B.P., Pochelon A. and Scarabosio A. 2006 Phys. Rev. Lett. 97235003

[10] McDermott R.M. et al 2011 Plasma Phys. Control. Fusion 53124013

[11] McDermott R.M., Angioni C., Conway G.D., Dux R., Fable E., Fischer R., Pütterich T., Ryter F., Viezzer E. and the ASDEX Upgrade Team 2014 Nucl. Fusion 54043009

[12] White A.E. et al 2013 Phys. Plasmas 20056106

[13] Rice J.E. et al 2011 Nucl. Fusion 51083005

[14] Luxon J.L. 2002 Nucl. Fusion 42614

[15] Mordijck S. et al 2012 Phys. Plasmas 19056503

[16] Takenaga H., Nagashima K., Sakasai A., Oikawa T. and Fujita T. 1998 Plasma Phys. Control. Fusion 40 183-90

[17] Jakubowski M.W. et al 2013 Nucl. Fusion 53113012

[18] Salmi A. et al 2014 Gas puff modulation experiments in JET L- and H-mode plasmas 41st EPS Conf. on Plasma Physics (Berlin, Germany) http://ocs.ciemat.es/EPS2014PAP/pdf/ P1.008.pdf

[19] O'Rourke J., Rimini F.G. and Start D.F.H. 1992 Nucl. Fusion 321861

[20] Gentle K.W., Gehre O. and Krieger K. 1992 Nucl. Fusion 32217

[21] Lopes N.J. 1995 Plasma Phys. Control. Fusion 37799

[22] Jacchia A., Mantica P., De Luca F. and Gorini G. 1991 Phys. Fluids B 3 3033-40

[23] Salmi A. et al 2015 Particle source and edge transport studies in JET H-mode gas puff modulation experiments 42nd EPS Conf. on Plasma Physics (Lisbon, Portugal) http://ocs.ciemat. es/EPS2015PAP/pdf/P2.135.pdf

[24] Staebler G.M., Kinsey J.E. and Waltz R.E. 2007 Phys. Plasmas 14055909

[25] Hillesheim J.C., Peebles W.A., Rhodes T.L., Schmitz L., Carter T.A., Gourdain P.-A. and Wang G. 2009 Rev. Sci. Instrum. 80083507

[26] Beurskens M.N.A. et al 2014 Nucl. Fusion 54043001

[27] Waltz R.E. and Miller R.L. 1999 Phys. Plasmas 64265 